\title{
Infections by Protozoa in Immunocompromised Hosts
}

\author{
Marcelo Simão Ferreira
}

\begin{abstract}
Disciplina de Doenças Infecciosas e Parasitárias, Faculdade de Medicina, Universidade Federal de Uberlândia, Rua Goiás 480/500, 38400-027 Uberlândia, MG, Brasil
\end{abstract}

\begin{abstract}
Protozoa are among the most important pathogens that can cause infection in immunocompromised patients. They infect particularly individuals with impaired cell immunity, such as those with hematologic neoplasias, those submitted to transplant of solid organs, those under high-dose corticosteroid therapy, and carriers of the human immunodeficiency virus. Among the protozoa that most commonly cause disease in immunocompromised individuals are Toxoplasma gondii, Trypanosoma cruzi, Cryptosporidium parvum, Isospora belli, Cyclospora cayetanensis and microsporidia; the former two cause severe encephalitis and myocarditis, and the others cause gastrointestinal infections. Early diagnosis and prompt institution of specific therapy for each of these organisms are basic measures to decrease morbidity and mortality associated with these infections.
\end{abstract}

Key words: protozoa - immunosupression - acquired immunodeficiency syndrome-Aids - opportunistic infections

A great number of protozoa may infect man. These unicellular beings often present complex evolutionary cycles and, contrary to what occurs with most helminths have the ability to replicate and adapt in their hosts. This phenomenon explains their long survival in various organs, even in the absence of clinical symptoms. Many protozoa such as those that cause malaria and leishmaniasis, depend on the action of vectors to be transmitted to man but in others, transmission is independent of the intermediate hosts' actions, as seen in amebiasis and giardiasis. Many of these parasites behave as opportunists, leading to disease mainly in those who host illnesses that suppress cellular immunity. Examples of such illnesses are: the infection caused by the human immunodeficiency virus (Aids) and lymphoproliferative diseases. Those who have had transplants (kidney, liver, bone marrow, etc.) and have been submitted to immunosuppressive therapy, collagen vascular diseases that use corticosteroids, among others, also have their cellular immunity suppressed and therefore are susceptible to opportunistic infections.

Various protozoa have been incriminated for causing disease in the immunosuppressed. Among them are Toxoplasma gondii, Trypanosoma cruzi, Leishmania sp., Cryptosporidium parvum, Isospora belli, microsporidia (various species), Giardia lamblia, Cyclospora cayetanensis, and less fre-

Fax: +55-31-263.3151

Received 7 August 2000

Accepted 4 September 2000 quently Babesia sp. Malarial parasites, Entamoeba histolytica, Trichomonas vaginalis and Balantidium coli do not have opportunistic behavior. Pneumocystis carinii is not considered as a protozoan but it is classified among the fungus and therefore will not be commented upon. Exceptionally, free living amebae (Acanthamoeba sp.) can cause disease in the immnosuppressed. Meningoencephalitis is a prime example (Martinez 1980).

$T$. gondii, $T$. cruzi, and $L$. chagasi may cause latent endogenous infections. When there is sufficient severe suppression of the immune response these pathogens trigger a severe form of the disease which in most individuals is, in general, systemic and fatal, especially if there is no early diagnosis and no adequate specific therapy. Some intestinal protozoa can also present opportunistic behavior ( $C$. parvum, I. belli) but the acquisition of the pathogens is exogenous, by oral-faecal route, and the clinical symptoms show greater severity in the immunosuppressed (Luft \& Remington 1992, Rosenthal et al. 1995, Ferreira et al. 1997).

$T$. gondii is recognized as one of the most important protozoa which afflicts immunocompromised hosts. Most frequently, the infection results in reactivation of latent chronic infections from cysts that are present in the brain, eyes, heart and muscles. Severe primary infections also occur in those who are immunodeficient. Patients with Aids are the ones most affected by the infection, although those who have lymphomas, have received transplants and have chronic inflammatory diseases of the connective tissues can also be afflicted by this pathogen. Focal meningoencephalitis mainly attacks basal ganglia and is the most common clinical manifestation observed in the immunosup- 
pressed. Nevertheless, myocarditis, intersticial pneumonitis and disseminated disease may also occur in these individuals (Luft \& Remington 1992). Serum IgM antibodies are rarely present (except in primary infections) and many times, diagnosis may only be made based on anatomopatological findings. Imaging methods (nuclear magnetic resonance, computerized axial tomography) may reveal focal lesions in the nervous system, although the images are very similar to abscesses, tumors and other opportunistic infections of the nervous system (Ciricillo \& Rosenblum 1990). Combination of sulfadiazine (or clindamycin) and pyrimetamine represents the most effective initial therapy in the treatment of this protozoan disease. Treatment should extend from four to six weeks and secondary prophilaxis should be recommended to avoid further reactivation (Porter \& Sande 1992).

Protozoans of the genus Leishmania may also present opportunistic behavior. Cases of mucocutaneous or visceral leishmaniasis have been reported more and more to be associated with immunosuppressive disorders, mainly with the Aids. (Fernandez-Guerrero et al. 1987, Rosenthal et al. 1995). HIV-Leishmania co-infection has been a recognized problem in various Mediterranean and African countries and to a lesser extent in Latin American countries. In Southern Europe, more than a thousand cases of Aids associated visceral leishmaniasis have been reported and nowadays, around $70 \%$ of those who have this parasitic infection in Europe are co-infected with HIV. In Brazil a little more than fifty cases of mucocutaneous and visceral leishmaniasis associated with Aids have been described until now, but it is possible that this number will increase as urbanization of Leishmania infections occurs (Rabello et al. 1998). Similar to what occurs in toxoplasmosis, clinical signs of the disease become evident in Aids patients when number of T CD4 lymphocytes is lower than $200 / \mathrm{mm}^{3}$. The majority of these infections undoubtedly represent reactivation of latent clinical infections. Regarding clinical aspects, the presence of skin ulcers and mucous membranes of varied severity are found in the mucocutaneous forms and the frequent presence of disseminated lesions is very similar to that observed in the diffuse classical form of the disease (Coura et al. 1987). Fever, splenomegaly and pancytopenia occur in approximately 75 to $80 \%$ of those with the visceral form and frequently other signs such as adenopathies, asthenia, weight loss, and hepatomegaly are present. Non typical localization of the disease is observed with a certain frequency in co-infected patients, particularly along the digestive tube, the respiratory tract, skin and central nervous system
(Rosenthal et al. 1995, Alvar et al 1997, Borges et al. 1999). The differential diagnosis should be made with other opportunistic infections that attack the Aids patient, principally histoplasmosis, miliary tuberculosis, and lymphomas. Diagnosis is based on the finding of parasite amastigotes in mucocutaneous biopsies, bone marrow and the spleen aspirates or through serological tests whose positivity is around $50 \%$. Culture of this material is mandatory for diagnosis because it permits the identification of the species in the process since the parasites that cause the cutaneous disease may be visceralized in co-infected patients. Response to initial treatment with pentavalent antimonials is around $50 \%$ and the reponse obtained with anfotericina B is $100 \%$. Relapse is common and a secondary prophylaxis with pentamidine or even with monthly-administered antimonials is recommended (Alvar et al. 1997).

Since the 60's, T. cruzi is known for its capacity to cause severe infections, particularly meningoencephalitis and myocarditis, in the immunocompromised. Cases of severe reactivated chagasic infections, frequently fatal, in patients with hematological neoplasias, kidney and heart transplants, and Aids have been described in the literature. Cases of acute primary T. cruzi infection are also rarely observed in immunosuppressed individuals who have received transfusion with contaminated blood with this protozoan (Grant et al. 1989, Cantarovich et al. 1992, Ferreira et al. 1997). In those who have had heart transplants and have had reactivated chagasic infection after the transplant because of the use of potent immunosuppressive drugs, meningoencephalitis is rare and the disease is manifested as severe myocarditis and subcutaneous lesions. It is rare to find the parasite in the peripheral blood. In kidney transplants, infection may be acquired through organ donation by an individual in the chronic form of the disease (Kirchoff 1993). In Aids patients, reactivation of Chagas disease is manifested as a feverish syndrome accompanied by meningoencephalitis and/ or acute myocarditis. A little more than 60 cases of HIV- T. cruzi co-infection have been recognized. The majority of these have occurred in Brazil and Argentina. A great part of these cases are in advanced stages of Aids with T CD4 lymphocyte counts lower than $200 / \mathrm{mm}^{3}$ and frequently with other opportunistic infections that define this syndrome. The central nervous system is involved in about $75 \%$ of the cases; meningoencephalitis is unifocal or multifocal and clinically presents itself with fever, headache, vomits, convulsions and focal signs. Cerebrospinal fluid exhibits a light pleocytosis with an increase in the number of lymphocytes and monocytes and raised protein levels. 
Computerized tomography and magnetic resonance show the presence of lesions generally located between the cortex and white matter. Frequently they have the shape of a ring - enhancing lesion or a dense nodule after contrast injection. An inflammatory infiltration is histologically observed in the presence of numerous $T$. cruzi amastigotes infecting the glial cells and rarely neurons. Immunohistochemical techniques are fundamental to confirm the parasite's identity. The heart is attacked less frequently (45\%) and myocarditis is difficult to be recognized in a clinical context. Arrhythmias and heart failure may occur due to myocardial involvement. Endocavitary biopsy, when available, may confirm the diagnosis. Direct detection of the parasite in the blood, pericardial fluid blood cultures and biopsies of infected tissues are the main ways to confirm diagnosis. Xenodiagnosis is not useful for diagnostic purpose since it is positive in more than $50 \%$ of chronic chagasic patients without Aids. Benznidazol and nifurtimox are both effective in the treatment of disease reactivation in the immunosuppressed. Benznidazol may be used in $5 \mathrm{mg} / \mathrm{kg}$ per day (in two doses) for 60 days. Side effects are common, especially dermatitis, agranulocytosis and peripheral neuropathy. Prognosis is not good and the majority die between 10 to 20 days after the initial diagnosis. Introduction of antiretroviral therapy in the treatment of Aids greatly decreased the number of cases of Chagas disease reactivation, thanks to the capacity of these drugs in maintaining T CD4 counts above 200 cells $/ \mathrm{mm}^{3}$ for long periods of time (Ferreira et al. 1991, 1997, Rocha et al. 1993, 1994).

In the last two decades, numerous protozoa have been identified as the cause of intestinal infection in immunosuppressed patients, particularly in those who have Aids. Among them we should mention C. parvum, I. belli, G. lamblia, microsporidia (Enterocytozoon bieneusi and Encephalitozoon intestinalis). All are acquired orally through contaminated food and water. In Aids patients, these protozoa produce chronic diarrhea leading to malabsorption, weight loss, and dehydration. Biliary tract infections caused by these pathogens have been reported in the literature, such as acute cholecystitis and sclerosing cholangitis, have already been observed predominately in the course of Cryptosporidium, Cyclospora and microsporidia infections. Diagnosis is based on the finding in stoool examination of Giardia cysts, Cryptosporidium, Isospora and Cyclospora oocysts and microsporidia spores. The combination of sulfametoxazol-trimethoprin is active upon Isospora and Cyclospora and albendazole is moderately effective in microsporidia infections. There is no therapy for Cryptosporidium. Paramomycin and macrolides are among the drugs that produce better results in cryptosporidiosis. Giardiasis is conveniently treated with metronidazole or tinidazole (Bello Sillo \& Gorbach 1998). Finally, Babesia microti and Acanthamoeba infections have been rarely observed in immunosuppressed patients. Babesia causes a severe and persistent disease in those who have had their spleens removed, but there are very well reported cases in Aids patients who present fever and hemolytic anemia. Quinine and clindamycin together seem to be effective in the treatment of this protozoosis. Free living amebae of the genus Acanthamoeba are rare causes of brain disease in Aids patients. These microorganisms may be isolated from water, soil, and air and generally cause granulamatous encephalitis that may be fatal in weeks to months. More than 20 of these cases have been reported in the literature. Disseminated disease that involves skin, paranasal air sinuses and nasopharynx has already been observed in Aids patients. Trophozoites and cysts may be found in the patient's CSF and brain tissue. The treatment for this infection is difficult and there is no secure effective therapeutic regimen.

\section{REFERENCES}

Alvar J, Canavate C, Gutierrez-Solar B, Jiménez M, Laguna F, López-Velez R, Molina R, Moreno J 1997. Leishmania and human immunodeficiency virus coinfection: the first 10 years. Clin Microbial Rev 10: 298-318.

Bello Sillo NA, Gorbach SL 1998. Diarrhea and HIV infection. Inf Dis Clin Pract 7: 213-219.

Borges AS, Machado AA, Ferreira MS, Figueiredo JF, Silva GF, Cimerman S, Bacha HA, Teixeira MCL 1999. Concomitância de leishmanioses e infecção pelo vírus da imunodeficiência humana (HIV): estudo de quatro casos. Rev Soc Bras de Med Trop 32: 713-719.

Cantarovich F, Vazquez M, Duro Garcia W, Abbud Filho M, Herrera C, Hernandez AV 1992. Special infections in organ transplantation in South America. Transpl Proc 24: 1902-1908.

Ciricillo SF, Rosenblum ML 1990. Use of CT and MR imaging to distinguish intracranial lesions and to define the need for biopsy in AIDS patients. $J$ Neurosurg 74: 720-724.

Coura JR, Galvão-Castro B, Grimaldi G 1987. Disseminated American cutaneous leishamniasis in a patient with AIDS. Mem Inst Oswaldo Cruz 82: 581-582.

Fernandez-Guerrero ML, Aguado JM, Buzon L, Barros C, Montalban C, Martin T, Bouza E 1987. Visceral leishmaniasis in immunocompromised hosts. Am J Med 83: 1098-1102.

Ferreira MS, Nishioka AS, Rocha A, Silva AM, Ferreira RG, Olivier W, Tostes Jr S 1991. Acute fatal Trypanosoma cruzi meningoencephalitis in a human immunodeficiency virus - positive hemophiliac patient. Am J Trop Med Hyg 45: 723-727. 
Ferreira MS, Nishioka SA, Silvestre MTA, Borges AS, Nunes-Araújo FRF, Rocha A 1997. Reactivation of Chagas' disease in patients with AIDS: report of three new cases and review of the literature. Clin Infect Dis 25: 1397-1400.

Grant IH, Gold JWM, Wittner M, Tamowitz HB, Nathan C, Mayer K, O'reilly RJ, Armstrong D 1989. Transfusion-associated acute Chagas' disease acquired in the United States. Ann Intern Med 111: 849-851.

Kirchoff LV 1993. American trypanosomiasis (Chagas' disease) a tropical disease now in the United States. New Engl J Med 329: 639-644.

Luft BJ, Remington JS 1992. Toxoplasmic encephalitis in AIDS. Clin Infect Dis 15: 211-222.

Martinez AJ 1980. Is Acanthamoeba encephalitis an opportunistic infection. Neurology 30: 567-574.

Porter SB, Sande MA 1993. Toxoplasmosis of the central nervous system in the acquired immunodeficiency syndrome. N Engl J Med 329: 995-1000.

Rabello A, Andrade MO, Disch J 1998. Co-infecção
Leishmania/HIV. Rev Soc Bras Med Trop 31 (Supl. III): 81-91.

Rocha A, Ferreira MS, Nishioka SA, Silva M, Burgarelli MKN, Silva AM, Moura LP, Ugrinovich R, Raffin C 1993. Trypanosoma cruzi meningoencephalitis and myocarditis in a patient with acquired immunodeficiency syndrone. Rev Inst Med Trop São Paulo 35: 205-208.

Rocha A, Menezes ACO, Silva AM, Ferreira MS, Nishioka AS, Burgarelli MKN, Almeida E, Turcato Jr G, Metze K, Lopes ER 1994. Pathology of patients with Chagas' disease and acquired immunodeficiency syndrome. Am J Trop Med Hyg 50: 261268.

Rosenthal E, Marty P, Poizot-Martin I, Reynes J, Pratling F, Lafemillade A, Jambert D, Boulat O, Dereure J, Gambarelli F, Gastant JÁ, Dujardin P, Dellamonica $\mathrm{P}$, Cassuto JP 1995. Visceral leishmaniasis and HIV1 co-infection in southern France. Trans $R$ Soc Trop Med Hyg 89: 159-162. 